\title{
A STABILITY THEOREM ON QUASI-REFLEXIVE OPERATORS
}

\author{
T. C. WU
}

\begin{abstract}
A range-closed bounded linear operator between Banach spaces is quasi-reflexive if both its kernel and cokernel are quasi-reflexive spaces. Under suitable conditions, if an operator is sufficiently close to a quasireflexive operator, it is itself quasi-reflexive.
\end{abstract}

1. Introduction. Let $X$ and $Y$ be Banach spaces. In [1], Civin and Yood defined that $X$ is quasi-reflexive of order $n$ if $X^{* *} / J(X)$ is of finite dimension $n$, where $X^{* *}$ is the second conjugate space of $X$ and $J: X \rightarrow X^{* *}$ is the natural injection. A quasi-reflexive space of order 0 is simply a reflexive space. Let $B(X, Y)$ be the Banach space of bounded linear operators from $X$ to $Y$. An operator $T \in B(X, Y)$ is called quasi-reflexive of type $(m, n)$ if it has closed range and if $\operatorname{Ker}(T)$ and $\operatorname{Coker}(T)$ are quasi-reflexive of order $m$ and $n$, respectively. In [3], such operators are called generalized Fredholm operators when the kernels and cokernels are both reflexive spaces, and a rather extensive theory for such operators is developed. We shall prove that under suitable conditions, if an operator is sufficiently close to a quasi-reflexive operator, it is itself a quasi-reflexive operator; and if $\phi(T)=m-n$ for a quasi-reflexive $T$ of type $(m, n)$, and $S$ is close to $T$, then $\phi(S)=\phi(T)$.

2. An auxilliary lemma. Recall that a closed subspace of $X$ splits if it has a closed complementary subspace in $X$. We consider the following two conditions on Banach spaces:

(C') Every quasi-reflexive subspace splits.

$\left(C^{\prime \prime}\right)$ Every closed subspace with quasi-reflexive quotient space splits.

LEMMA. Let $X$ be a Banach space satisfying condition $\left(\mathrm{C}^{\prime \prime}\right)$. Let $M$ and $N$ be closed subspaces of $X$, and $M+N$ their vector sum. Assume that $M+N$ be closed and that $X / M$ is quasi-reflexive. Then $M+N$ splits in $X$.

Proof. It is assumed that $M+N$ is a closed subspace of $X$. By $\left(\mathrm{C}^{\prime \prime}\right)$, we need only show that $X /(M+N)$ is quasi-reflexive. For a Banach space $Y$, let $\bar{Y}=Y^{* *} / J(Y)$. There is a natural onto map $p: X / M \rightarrow X /(M+N)$ which induces an onto map $\bar{p}: \overline{X / M} \rightarrow \overline{X /(M+N)}$. As $\overline{X / M}$ is finite dimensional, $\overline{X /(M+N)}$ is finite dimensional. Q.E.D.

Received by the editors September 28, 1976.

AMS (MOS) subject classifications (1970). Primary 47B05.

Key words and phrases. Banach spaces, complementary subspace, quasi-reflexive, double-splitting, kernel, cokernel. 
3. The Theorem. It is easy to see that if $X$ is quasi-reflexive of order $n$, and $Y$ is isomorphic to $X$, then $Y$ is quasi-reflexive of order $n$. If $X$ is a closed subspace of $Y$, then $Y$ is quasi-reflexive of order $n$ if and only if $X$ and $Y / X$ are quasi-reflexive of order $m$ and $k$, respectively, and $n=m+k[1$, Corollary 4.2]. Recall that a bounded linear operator is called double-splitting if it has closed range and both its kernel and cokernel split.

THEOREM. Let $T \in B(X, Y)$ be double-splitting and quasi-reflexive of type $(m, n)$. Assume that $X$ and $Y$ satisfy condition $\left(C^{\prime \prime}\right)$. Let $S \in B(X, Y)$ be range-closed and sufficiently close to $T$. Then $S$ is double-splitting and quasireflexive of type $\left(m^{\prime}, n^{\prime}\right)$ with $m^{\prime}-n^{\prime}=m-n$.

Proof. Write $X=\operatorname{Ker}(T) \oplus M$ and $Y=T(X) \oplus N$, where $M$ and $N \cong$ Coker $(T)$ are closed complementary subspaces of $\operatorname{Ker}(T)$ and $T(X)$, respectively. Following [2, Theorem 4, p. 122], let $p: M \oplus N \rightarrow M$ and $q: M \oplus N$ $\rightarrow N$ be the projections. Consider the map $f(S)=S \cdot p+q: M \oplus N \rightarrow Y$, for $S \in B(X, Y)$. Then $f$ is a continuous map from $B(X, Y)$ into $B(M \oplus$ $N, Y)$. By the open mapping theorem, $f(T)$ is an isomorphism, hence $f(S)$ is an isomorphism and $Y=f(S)(M) \oplus f(S)(N)$ for $S$ close to $T$. But $f(S)(M)$ $=S(M)$ and $f(S)(N)=N \cong \operatorname{Coker}(T)$, so that $Y \cong S(M) \oplus \operatorname{Coker}(T)$.

Since $S$ is one-to-one on $M, \operatorname{Ker}(S) \oplus M=\operatorname{Ker}(S)+M$, and since $X / M$ $=\operatorname{Ker}(T)$ is quasi-reflexive, $\operatorname{Ker}(S) \oplus M$ has a closed complementary subspace $V$ in $X$ by the Lemma, and then

$$
X=\operatorname{Ker}(S) \oplus M \oplus V=\operatorname{Ker}(T) \oplus M .
$$

Thus $\operatorname{Ker}(S)$ splits in $X$ and $\operatorname{Ker}(S) \oplus V=\operatorname{Ker}(T)$. As $\operatorname{Ker}(T)$ is quasireflexive, $\operatorname{Ker}(S)$ and $V$ are quasi-reflexive. By assumption, $S(X)$ is closed, and since $S: M \oplus V \rightarrow S(X)$ is bijective and continuous, $S$ is an isomorphism from $M \oplus V$ onto $S(X)$. Therefore $S(V)$ is closed and isomorphic to $V$ so that $S(V)$ is quasi-reflexive. It is clear that $S(M)$ is closed and has a quasi-reflexive complementary subspace in $Y$, and the image $S(X)=S(M)+S(V)$ of $S$ splits in $Y$ by the Lemma. We have proved that $S$ is double-splitting. Thus

$$
Y \cong S(M) \oplus S(V) \oplus \operatorname{Coker}(S) \cong S(M) \oplus \operatorname{Coker}(T)
$$

and $\operatorname{Coker}(T) \cong \operatorname{Coker}(S) \oplus S(V)$. It follows that $\operatorname{Coker}(S)$ is quasi-reflexive and therefore $S$ is quasi-reflexive. Since $\operatorname{Ker}(T) \cong \operatorname{Ker}(S) \oplus V$, $\operatorname{Coker}(T) \cong \operatorname{Coker}(S) \oplus S(V)$ and $V \cong S(V)$, we have $m^{\prime}-n^{\prime}=m-n$. Q.E.D.

We notice that if $X$ satisfies condition $\left(\mathrm{C}^{\prime}\right)$, and $Y$ satisfies condition $\left(\mathrm{C}^{\prime \prime}\right)$, then any quasi-reflexive operator is double-splitting.

COROllary. Let $X$ be a Banach space satisfying $\left(\mathrm{C}^{\prime}\right)$ and $\left(\mathrm{C}^{\prime \prime}\right)$, and $Y$ a Banach space satisfying $\left(\mathrm{C}^{\prime \prime}\right)$. Let $T \in B(X, Y)$ be quasi-reflexive of type $(m, n)$. If $S \in B(X, Y)$ is range-closed and close to $T$, then $S$ is quasi-reflexive of type $\left(m^{\prime}, n^{\prime}\right)$ with $m^{\prime}-n^{\prime}=m-n$. 
4. Remarks. If we replace 'quasi-reflexive' in conditions $\left(\mathrm{C}^{\prime}\right)$ and $\left(\mathrm{C}^{\prime}\right)$ by 'reflexive', we have a corresponding theorem for generalized Fredholm operators as defined in [3]. If we consider only finite dimensional subspaces, condition $\left(C^{\prime}\right)$ and $\left(C^{\prime \prime}\right)$ are void, the Theorem assumes a much stronger form and gives the result for classical Fredholm operators [2, Theorem 4, p. 122].

Let $\phi(T)=m-n$ for a quasi-reflexive operator $T$ of type $(m, n)$. If $T \in B(X, Y)$ and $S \in B(Y, Z)$ are quasi-reflexive operators and $S T \in$ $B(X, Z)$ is range-closed, then $S T$ is also quasi-reflexive and $\phi(S T)=\phi(S)+$ $\phi(T)$. In fact, if one employs the functor $-: B \rightarrow B$ in [3], one can show that a range-closed operator $T \in B(X, Y)$ is quasi-reflexive if and only if $\bar{T} \in$ $B(\bar{X}, \bar{Y})$ is Fredholm, and then $\phi(T)=\operatorname{ind}(\bar{T})$, the index of $\bar{T}$.

\section{REFERENCES}

1. P. Civin and B. Yood, Quasi-reflexive spaces, Proc. Amer. Math. Soc. 8 (1957), 906-911.

2. R. S. Palais et al., Seminar on the Atiyah-Singer index theorem, Ann. of Math. Studies, no. 57, Princeton Univ. Press, Princeton, N. J., 1965.

3. K. W. Yang, The generalized Fredholm operators, Trans. Amer. Math. Soc. 216 (1976), 313-326.

765 Amsterdam Avenue, New York, New York 10025 